\section{AB0970 INVESTIGATION OF THE EFFECTS OF BALANCE TRAINING ON BALANCE AND FUNCTIONAL STATUS IN PATIENTS WITH TOTAL HIP ARTHROPLASTY DUE TO OSTEOARTHRITIS: A RANDOMISED CONTROLLED PILOT STUDY}

N. Elibol ${ }^{1}$, B. Unver ${ }^{2}$, V. Karatosun ${ }^{3} .{ }^{1}$ Physiotherapy and rehabilitation, Europian of Lefke University; ${ }^{2}$ Physiotherapy and rehabilitation; ${ }^{3}$ Orthopaedics, Dokuz Eylul University, Izmir, Turkey

Background: Osteoarthritis (OA) of the hip is one of the most common disorders in musculoskeletal system. The hip osteoarthritis is painful and this pain causes disability of various degrees, postural and gait disorders. ${ }^{1}$ Total hip arthroplasty (THA) has been to one of the most frequent elective surgical procedures that can effectively reduce pain and improve the function in patient with hip OA. ${ }^{2}$ It was reported that patients with coxarthrosis and THA have decreased proprioception with motor control and balance disorders, compared to healthy subjects. ${ }^{3}$

The purpose of current study was to investigate the effects of balance exercises on balance and functional level with objective assessment methods until the 26th week of surgery in patients with THA.

Objectives: The purpose of our study is investigating of the effects of balance training on balance and functional status in patients with THA.

Methods: Sixteen patients with unilateral elective THA were randomised to 2 groups: conventional rehabilitation ( $\mathrm{CR}, \mathrm{n}=8)$ or conventional rehabilitation plus balance training $(C R+B T, n=8)$ groups. The $C R$ group completed typical surgery-specific joint range-of-motion and muscle strengthening exercises, while the $\mathrm{CR}+\mathrm{BT}$ group completed the CR plus balance exercises during 6 weeks. The patients were evaluated by single leg stance test, Tetrax balance system, Harris hip scoring, lower extremity function scale, 5 times sit to stand test and 50 -foot timed walk test preoperatively and 8,14 , and 26 weeks after THA.

Results: While there was significant improvement, in terms of on the right extremity eyes closed single leg stance test in the $C R$ group $(p<0.05)$, there was no significant difference in terms of other assessment parameters between $C R$ and $C R$ + BT groups $(p>0.05)$. There were significant improvement after THA surgery in both groups $(p<0.05)$.

Conclusions: The results of our study indicate that there were similar improvements in the balance and functional parameters in the $C R$ and $C R+B T$ groups. There was no additional benefit of the balance exercises in balance in the 14 and 26 weeks after THP. Significiant differences could be found between groups by continuing balance training with more patients for $1-2$ years following THA.

\section{REFERENCES:}

[1] Truszczynska A, Rapala K, Gmitrzykowska E, Trzaskoma Z, Drzal-Grabiec J. Postural stability disorders in patients with osteoarthritis of the hip. Acta Bioeng Biomech 2014;16 (1): 45-50.

[2] Sharma L. Osteoarthritis year in review 2015. Clinical Osteoarthr Cartil 2016;24 (1): 36-48.

[3] Moraes MR, Cavalcante ML, Leite JA, et al. The characteristics of the mechanoreceptors of the hip with arthrosis. J Orthop Surg Res. 2011;6 (1): 58.

Disclosure of Interest: None declared

DOI: 10.1136/annrheumdis-2018-eular.1591

\section{AB0971 COMBINATION OF HYALURONATE AND SODIUM SUCCINATE IN TREATMENT OF KNEE OSTEOARTHRITIS}

O. Burianov ${ }^{1}$, L. Khimion ${ }^{2}$, T. Omelchenko ${ }^{1}$, H. Havryliuk ${ }^{2} .{ }^{1}$ Traumatology and Orthopedics, Bogomolets National Medical University; ${ }^{2}$ Family Medicine, Shupyk National Medical Academy of Postgraduate Education, Kyiv, Ukraine

Background: At present hyaluronic acid $(\mathrm{HA})$ is rather widely used in treatment of patients with osteoarthritis $(\mathrm{OA})$. HA normalises the properties of the synovial fluid; has a protective effects; promotes the cartilage nutrition and so improves the signs of $\mathrm{OA}$ and function of the joints. To the contrary the effects of sodium succinate (the salt of the succinic acid) are not investigated but can be promising in $O A$ treatment due to its known anti hypoxic and energetic properties.

Objectives: To investigate the clinical efficacy of combination of hyaluronate and sodium succinate in $\mathrm{OA}$ treatment in early stages.

Methods: The study included 126 patients with knee OA (stages I-II, Kelgren and Lawrence), mean age $(54.3 \pm 2.7)$ years, among them -75 women $(60 \%)$ and 51 men $(40 \%)$. All enrolled patients had OA exacerbation (without clinically evident synivitis) and received standard OA treatment (NSAIDs, exercises, orthopaedic devices) for 15 days; Gr.1 patients (58) also got 5 intra-articular injections of $1.1 \%$ hyaluronic acid, stabilised with sodium succinate (2 $\mathrm{ml}$, once a week); patients of Gr.2 (68) in addition to standard treatment received 5 intra-articular injections of $1,1 \%$ solution of non-stabilised HA ( $2 \mathrm{ml}$, once a week). Clinical observation and evaluation of the results were performed at the beginning of the treatment, at 6th, 12th and 24th week after the study beginning

Results: During the treatment period, patients in both groups showed the positive changes in clinical signs and symptoms of OA. The VAS score in both groups indicated a significant pain reduction, but the stability and duration of the clinical effect in the groups was different. In patients of Gr.1, the pain syndrome continued to decrease after 12 weeks till 24th week, whereas in Gr.2 after the treatment course there was no significant changes in further pain regression after 6th week point The general WOMAC index was decreased from $(78.3 \pm 4.1)$ in Gr. 1 and $(75.4$ $\pm 3.8)$ in Gr. 2 at the beginning of the study to $(27.9 \pm 2.6)$ and $(29.8 \pm 1.9)$ accordingly at week $12, p<0,05$. The changes in Lisholm score were also significantly better in Gr. 1 than in Gr. 2 (before treatment $(21.7 \pm 4.6)$ and $(22.6 \pm 5.3)$, at week 6 $-(86.4 \pm 5.7)$ and $(71.3 \pm 4.8)$, at week $12-(87.6 \pm 6.2)$ and (63.8 \pm 5.3$)$, respectively, $\mathrm{p}<0,05$.

Conclusions: Combination of the hyaluronic acide and sodium succinate in early stages of knee OA (as intra-articular injections) allows to increase the treatment efficacy. Add of sodium succinate to hyaluronic acide achieve better pain control and longer remission.

Disclosure of Interest: None declared

DOI: 10.1136/annrheumdis-2018-eular.5487

\section{AB0972 THE EFFECTIVENESS OF PHYSICAL ACTIVITYINTERVENTIONS FOR PEOPLE WITH OSTEOARTHRITIS AND OBESITY: A META-ANALYSIS}

S. Mckevitt, C. Jinks, E.L. Healey, J.G. Quicke. Primary Care and Health Sciences, Keele University, Keele, UK

Background: Osteoarthritis $(\mathrm{OA})$ is one of the diseases with the highest prevalence of comorbidity. Clinical guidelines recommend physical activity (PA) for people with OA irrespective of comorbidity. Research investigating the effectiveness of PA interventions in OA and comorbidity is needed.

Objectives: To synthesise existing evidence investigating the effectiveness of PA interventions in adults with $O A$ and obesity.

Methods: A systematic review with meta-analysis was conducted (PROSPERO Registration: CRD42017055582). Six electronic databases; MEDLINE, EMBASE, AMED, CINAHL, SportDiscus and CENTRAL were searched for studies from their inception to 29.03.17. Inclusion criteria were: randomised controlled trials (RCTs) comparing the effectiveness of any PA intervention to non-PA control group; including adults aged 45 years old and over with clinical or radiographic $\mathrm{OA}$ at any site; at least one of the comorbidities of interest (COPD, depression, diabetes, hypertension, obesity, T2DM); and measuring pain, physical function, quality of life, global health post intervention and adverse events. Included study risk of bias (ROB) was assessed using the Cochrane risk of bias tool. Two reviewers screened titles, abstracts and full text articles, checked data extraction, and carried out ROB assessment. Random-effects model meta-analysis pooled outcomes from sufficiently homogeneous studies to calculate effect sizes (Standardised Mean Difference (SMD) with 95\% confidence interval (CI)). Metaanalysis findings of the $\mathrm{OA}$ and obesity subgroup are reported.

Results: The literature search retrieved 8171 citations of which 14 studies ( $n=4224$ participants) were included in the full review, with 9 ( $n=1382$ participants) analysed in the $\mathrm{OA}$ and obesity subgroup. PA interventions included: aquatic, aerobic, strengthening and functional activity; of 1-18 months in duration.

Four studies of $O A$ and obesity measuring either Western Ontario Osteoarthritis Index (WOMAC) pain, WOMAC function or Six Minute Walking Test (6MWT) and were included in three meta-analyses. Best estimates showed PA to improve WOMAC pain $(n=3$ studies; $n=547$ participants; $S M D=-0.09(95 \% \mathrm{Cl})-0.65$, 0.47 ), improve WOMAC function ( $n=3$ studies, $n=415$ participants; $S M D=-0.35$ $(95 \% \mathrm{Cl})-0.89,0.18)$ and the 6MWT $(\mathrm{n}=4$ studies, $\mathrm{n}=573$ participants; $\mathrm{SMD}=-0.93(95 \% \mathrm{Cl})-0.49,2.35)$. However, results were not statistically significant. There was substantial between-trial outcome heterogeneity $\left(I^{2} ;=89.4 \%\right.$ $(p=0.000) ; 77.5 \%(p=0.012) ; 97.8 \%(p=0.000)$; respectively); results should be interpreted with caution.

$\mathrm{ROB}$ domain judgements were generally either low or unclear. A small minority of judgements were at high risk of bias.

Conclusions: Best estimates suggest small beneficial effects of physical activity on WOMAC pain, WOMAC function and the 6MWT. Mixed effectiveness among individual RCTs was likely due to heterogeneous intervention types, intensity and duration.

Acknowledgements: SM is funded by a Keele University Acorn PhD studentship. JQ is funded by the NIHR Academic Clinical Lectureship in Physiotherapy, awarded as part of Professor Christian Mallen's NIHR Research Professorship (NIHR-RP-2014-026). CJ and EH are part funded by the NIHR Collaboration for Leadership in Applied Health Research and Care West Midlands (NIHR CLAHRC WM). The views expressed in this article are those of the author(s) and not necessarily those of the NHS, the NIHR, or the DOH and Social Care.

Disclosure of Interest: None declared

DOI: 10.1136/annrheumdis-2018-eular.6421 